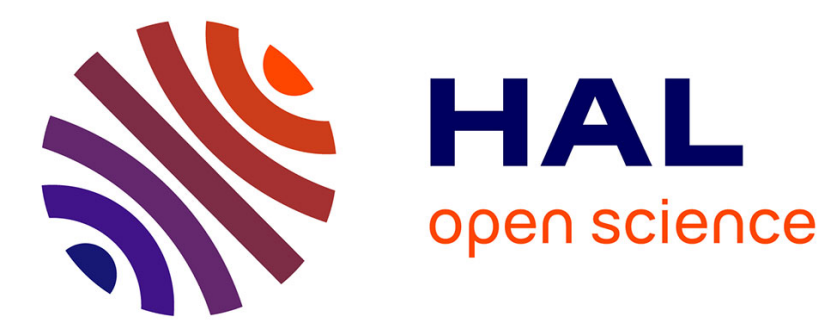

\title{
Laser Polymer Tattooing: A Versatile Method for Permanent Marking on Polymer Surfaces
}

Po-yi Chang, Adèle Bruntz, Loïc Vidal, Pierre-alain Vetter, Patrick Roudot, Laurent Bua, José L Ortiz, Hsiao-wen Zan, Olivier Soppera

\section{- To cite this version:}

Po-yi Chang, Adèle Bruntz, Loïc Vidal, Pierre-alain Vetter, Patrick Roudot, et al.. Laser Polymer Tattooing: A Versatile Method for Permanent Marking on Polymer Surfaces. Macromolecular Materials and Engineering, 2019, pp.1900402. 10.1002/mame.201900402 . hal-02323139

\section{HAL Id: hal-02323139 \\ https://hal.science/hal-02323139}

Submitted on 10 Nov 2020

HAL is a multi-disciplinary open access archive for the deposit and dissemination of scientific research documents, whether they are published or not. The documents may come from teaching and research institutions in France or abroad, or from public or private research centers.
L'archive ouverte pluridisciplinaire HAL, est destinée au dépôt et à la diffusion de documents scientifiques de niveau recherche, publiés ou non, émanant des établissements d'enseignement et de recherche français ou étrangers, des laboratoires publics ou privés. 


\section{WILEY-VCH}

OI: $10.1002 /(($ please add manuscript number $))$

Article type: Communication

\section{Laser polymer tattooing (LPT): a versatile method for permanent marking on polymer surfaces}

Po-Yi Chang ${ }^{a, b, c}$, Adèle Bruntz ${ }^{a, b}$, Lö̈c Vidal ${ }^{a, b}$ Pierre-Alain Vetter ${ }^{d}$, Patrick Roudot ${ }^{e}$, Laurent Bua $^{e}$, Jose Ortiz, Hsiao-Wen Zan ${ }^{c}$, Olivier Soppera ${ }^{a, b} *$

P.-Y. Chang, A. Bruntz, L. Vidal, O. Soppera

Université de Haute-Alsace, CNRS, IS2M UMR 7361, F-68100 Mulhouse, France

Université de Strasbourg, France

E-mail: Olivier.soppera@uha.fr

Prof. Hsiao-Wen Zan,

Department of Photonics and Institute of Electro-Optical Engineering, National Chiao Tung University, Hsinchu, Taiwan 30010, Republic of China

Pierre-Alain Vetter

IREPA Laser, 67400 Ilkirch, France

Patrick Roudot, Laurent Bua

Tiflex, 01450 Poncin, France

Jose Ortiz

Valeo Visibility, 93012 Bobigny, France

Keywords: Inkjet, nanoparticles, laser, polymer

\section{Abstract}

A versatile and efficient method for the permanent marking of polymer surfaces that combines inkjet deposition and near-infrared (NIR) laser curing is investigated. The NIR laser treatment forces the ink particles to migrate into the upper layers of the polymer. This results in the fixation of a permanent grayscale image that can be applied to various polymers, such as polypropylene, which is widely used in industry but still difficult to mark. The physiochemical processes induced by laser curing are investigated by electronic and optical microscopy. The dependence of the thickness of the deposited ink and the laser power on the 


\section{WILEY-VCH}

contrast of marking are also studied. A mechanism implying fast laser-induced melting of the polymer surface followed by displacement of carbon nanoparticles by convection is proposed. Finally, a comparison of the aging properties of samples prepared by our process and standard $\mathrm{UV}$ ink is proposed to illustrate the interesting nature of this new polymer marking process. Integrating the ink under the surface of the polymer, as in a skin tattooing procedure, by laser curing is an efficient way to generate permanent images on polymer surfaces.

In the manufacturing of polymers, marking is an important final step with high added value. Many manufactured polymer parts have markings for different purposes, such as decorating or personalizing objects, writing serial numbers or bar codes for identification, quality control, inscribing logo or product names, in both practical and cosmetic contexts. In most applications, the requirements are fast writing speed, writing directly on the finished product to avoid additional time-consuming and/or expensive steps, a reduced use of toxic or hazardous chemicals and ideally simple and versatile equipment. The process should also be easily digitalized to allow the direct writing of complex arbitrary shapes from a computergenerated file.

Inkjet printing has been increasingly used for polymer marking since the early 2000s.[1] However, this technique does not provide permanent marking due to the difficulty of ensuring good adhesion of the cured ink to the raw polymer surface. For instance, polyolefins require a specific surface treatment and an additional protective coating on top of the marking.

UV-curable inks were designed to improve the durability of markings in aggressive environments. UV curing is based on a photoinduced crosslinking reaction that solidifies the deposited ink. UV irradiation offers many advantages as it is fast and energy-efficient and limits the use and release of toxic solvents. The recent development of low-cost high-power 


\section{WILEY-VCH}

UV-LED light sources has increased industrial interest in such techniques. UV inkjets also allow the design of color drawings and deposition of functional materials for applications in biology[2] or electronics,[3] which is impossible with many other marking processes. However, UV inks present a major limitation in terms of adhesion and abrasion resistance, since the marking layer is added on top of the polymer surface and the adhesion of the cured inked to the polymer surface is thus crucial. Alternatively, e-beam curing can be used, but this technique requires sophisticated expensive equipment and is thus limited to very specific applications.

In this context, polymer marking by lasers has gained great attention during the last decade.[4] The technique is fast, cost-effective and versatile. It allows permanent and resistant marking. Marking is achieved by focusing a laser (usually a focused Nd-YAG pulsed laser emitting at $1064 \mathrm{~nm})[5,6,7]$ at the surface of a polymer. The beam is displaced at the surface by galvanometric mirrors, allowing fast writing (a few $\mathrm{m} / \mathrm{s}$, which makes it possible to write more than 100 alphanumeric characters per second). The minimum pixel size is typically 25 microns, which makes it possible to obtain well-defined figures.[8] With this technique, no ink is used, but the laser modifies the polymer surface to create an image pixel by pixel. Depending on the nature of the polymer and the laser power, several mechanisms can be involved. In this case, marking is the result of carbonization of the polymer surface under high laser power. At lower power, foaming or melting can be observed.[9,10] Most of the commonly used polymers are compatible with this marking method.[11] However, to guarantee an efficient process, the polymer needs to exhibit significant absorption at the laser wavelength. Poly(ethylene terephthalate) (PET), polycarbonate (PC), and polystyrene (PS) intrinsically exhibit some photosensitivity at $1064 \mathrm{~nm}$ and can be easily carbonized at high temperature so they can be marked without the use of additives.[12] However, even for these polymers, additives are usually added to increase the photosensitivity needed to reach a writing speed suitable for practical applications. Some polymers, such as polyolefins, are 


\section{WILEY-VCH}

transparent in the NIR region $(1000$ to $1100 \mathrm{~nm})$.[13,14] In this case, additives are required. These compounds can be either organic dyes (cyanine), metal complexes (copper complexes) or nano/microparticles (e.g., $\mathrm{TiO}_{2},[15,16] \mathrm{Sb}_{2} \mathrm{O}_{3}$, [17] $\mathrm{SnO}_{2}: \mathrm{Sb}_{2} \mathrm{O}_{3},[18] \mathrm{BiO}_{2}$, [15,19] black carbon,[20,21] and graphene[22]). Though the concentration of additives remains at a very low level (typically from 0.01 to $3.0 \mathrm{wt}$ \%), the additives have to be included in the bulk polymer before the fabrication of the polymer part. Thus, they increase the complexity and cost of polymer development and may have undesired consequences on the final material properties. In particular, the dispersion of the absorber must be very homogeneous in the polymer matrix. Moreover, the thickness of the region in which the polymer and laser interact can reach 100 microns for marking with good contrast. For thin samples or applications where the mechanical properties of the material are important (aeronautic, health, automotive), it is necessary to check the properties of the objects after marking, which limits the versatility of the process.

Laser deposition is also involved in laser-induced forward transfer (LIFT), which has very interesting features for locally depositing materials.[23] The nozzle-free setup in LIFT allows the use of highly viscous and/or functional inks with micrometric resolution.[24,25,26,27,28] However, in LIFT, the deposited material is transferred by a laser but remains at the surface of the substrate. Consequently, LIFT generally leads to low adhesion or abrasion properties. In this context, a process that combines aspects of both the inkjet approach and laser curing was developed.[29] In the process, ink is deposited by an inkjet on a polymer surface. Laser irradiation is then focused on the ink to make it enter the upper polymer layer. After laser curing, pigments are thus inserted in the polymer, which guarantees the resistance of the marking, as shown later. The key point is the control of the local thermal effect induced by the NIR laser. The objectives of this paper are to investigate the processes involved and demonstrate the interesting aspects of the concept. 


\section{WILEY-VCH}

\section{Results and discussion}

The principle of laser polymer tattooing (LPT) is illustrated in Figure 1a. The image to print is first generated on a computer and sent to a conventional printer head. Ink is deposited at the desired location by an inkjet, and the NIR laser stabilizes the ink. The NIR laser diode is installed in close vicinity to the inkjet nozzles. The deposition and fixation process are thus achieved in a single pass. The laser power is adjusted according to the ink deposition speed to deliver the needed energy to activate the process. One of the interesting aspects of this approach is that there is no excess ink to eliminate from the polymer surface. Indeed, as shown later, with an optimized ink thickness and laser power, it is possible to fix all of the deposited ink into the polymer substrate. In this work, the ink is a dispersion of pigment Black 7 in diethylene glycol diethylether. The concentration of the black dye in the ink is $5 \mathrm{wt} \%$. The polymer substrate is held on a translation stage and displaced during inkjet marking and laser curing at a typical speed of $5 \mathrm{~m} / \mathrm{min}$. The schematic in Figure $1 \mathbf{b}$ provides details on the different parts of the setup, including 1) the translation stage that moves the sample, 2) the inkjet nozzles, 3) the ink tanks, 4) electronic control of the inkjet heads and 5) the NIR laser diode $(808 \mathrm{~nm})$. An example of a gray-level image printed by LPT is given in Figure 1c. In this case, the resolution of the image was 180 dpi. The gray level was controlled via control of the drop volume and pixel density. A polypropylene (PP) substrate was used here. The polymer surface was only cleaned with ethyl acetate before printing, without any other specific surface treatment.

To investigate what happens to the black carbon nanoparticles during laser irradiation, transmission electron microscopy (TEM) characterizations were conducted. In this set of experiments, to simplify the sample preparation, ink was deposited by spin-coating on PP substrates. Here, spin-coating allows deposition of a continuous layer of ink with a thickness that is directly linked to the rotation speed during deposition. The laser irradiation conditions 


\section{WILEY-VCH}

were $5 \mathrm{~m} / \mathrm{min}$ at $60 \mathrm{~W}$. A thin section of the sample was then prepared by ultramicrotomy and observed by TEM. The results are shown in Figure 2. Figure 2a shows the first section of the raw polypropylene surface used in this study as a reference. In the sample shown in Figure $\mathbf{2 b}$, laser treatment $(5 \mathrm{~m} / \mathrm{min}, 60 \mathrm{~W})$ was conducted at the surface of the polymer, without any ink in this case. The morphology of the polymer was not modified by the laser. This is expected from the low absorption of PP at $808 \mathrm{~nm}$, so the laser beam passes through the sample without any significant interaction, preserving the smooth surface. It is worth noting that none of the other polymers used in this study showed any significant intrinsic interaction with the NIR laser under the conditions needed to cure the ink. This justifies the choice of wavelength and the laser irradiation parameters. Indeed, it is important that the parts of the sample that are not covered by the ink do not interact with the laser to keep the polymer properties intact. Under such conditions, laser irradiation only needs to be performed on the areas covered by ink, which simplifies the laser irradiation step. Figure 2c shows a TEM cross-section after deposition by spin-coating. A section of the uniform ink layer can be easily visualized on top of the PP surface. The thickness of this layer was evaluated to be approximately $400 \mathrm{~nm}$. Single black carbon nanoparticles are visible. As expected, they exhibit a wide polydisperse size distribution, with diameters ranging from 10 to $60 \mathrm{~nm}$. Figure $2 d$, Figure $2 \mathbf{e}$ and Figure 2f show the impact of the laser treatment on the PP surface covered with ink. The following can be observed. i) There is no longer ink on the surface of the polymer. ii) Carbon black nanoparticles can be observed inside the PP under the surface. Interestingly, the particles form large continuous aggregates with a width comparable to the initial thickness of the ink layer. This suggests that the ink layer initially present at the surface was transported by blocks inside the polymer. iii) The roughness at the polymer surface is significantly increased by the laser irradiation. iv) A separation line parallel to the surface (dashed line in Figure 2d) is observed. The depth of this line is approximately $2000 \mathrm{~nm}$. Such a separation was not observed in the polymer (Figure 2a and Figure 2b), even after laser irradiation. The difference in the contrast 


\section{WILEY-VCH}

between the 2 zones in the TEM image reveals a difference in the density induced by laser melting.

Based on these observations, it is possible to propose a mechanism explaining the ink/laser/polymer interaction: NIR laser irradiation is absorbed by the $\mathrm{C}$ nanoparticles of the ink layer, which causes a significant increase in temperature. Indeed, as shown in Figure S1, a 400 -nm-thick film absorbs $27 \%$ of the NIR light. The increase in temperature is high enough to provoke melting of the upper part of the polymer. We could not conduct a direct measurement of the temperature increase, but the observation by TEM of a change in density in the upper layer of the polymer proves that the first 2 microns under the surface of the PP melted. This is also supported by the presence of the carbon black nanoparticles under the surface of the PP. Obviously, the nanoparticles could not diffuse without melting of the PP. The temperature reached under laser irradiation is thus between $160{ }^{\circ} \mathrm{C}$ (fusion temperature measured by DSC, see Supporting Information, Figure S2) and $250{ }^{\circ} \mathrm{C}$, which is the degradation temperature of PP. The heating effect of the $\mathrm{C}$ nanoparticles is known and has already been reported in other contexts.[30,31,32] The heating of the polymer under laser irradiation to fusion temperature is in agreement with previous results from Fukumura et al. $\left[{ }^{33},{ }^{34},{ }^{35},{ }^{36}\right]$ The curved shape of the carbon nanoparticle aggregates strongly suggests that the nanoparticle movement was induced by the convective movement of the melted polymer. Photothermal convection was recently proposed as a driving force for the directed selfassembly of colloidal plasmonic nanoparticles on surfaces.[37] In our case, the main difference is that the nanoparticles are already immobilized on the surface, but the heat generated by the nanoparticles can also provoke the melting of the polymer. High-temperature gradients are created, inducing convective movements when the polymer upper layer is in a liquid state. The convective movement of the melted polymer drives the displacement of the $\mathrm{C}$ nanoparticles in a continuous movement that explains the observation of blocks of nanoparticles inside the polymer. Since the viscosity of the melted polymer is high, the 


\section{WILEY-VCH}

displacement of the $\mathrm{C}$ nanoparticles by diffusion cannot be proposed as a displacement mechanism. Photonic forces may also be invoked due to the strong absorption of the $\mathrm{C}$ nanoparticles that can result in repulsive forces applied to the $\mathrm{C}$ nanoparticles. It is difficult to state on the relative contribution of such forces in our experimental conditions, especially because the medium remains quite viscous. However, they are too weak to induce displacement in this viscous medium, and they would likely create a more chaotic distribution of the $C$ nanoparticles inside the polymer matrix. The melted polymer convection contribution is also supported by additional experiments performed with a fixed laser source in equivalent irradiation conditions. In that case, we observed heating of the $\mathrm{C}$ nanoparticle film, but it did not result in the displacement of the ink into the polymer. This showed that the movement of the laser head over the sample played a role in the displacement of the nanoparticles. A schematic is proposed in Figure $\mathbf{2} \mathbf{g}$ to summarize the nanoparticle displacement process.

The impact of important parameters, such as ink thickness and laser power, was then investigated to confirm our proposed mechanism. The results are shown in Figure 3. In this section, the method was as follows. To obtain a homogeneous thin film, ink was deposited on PP circular substrates (diameter of $5 \mathrm{~cm}$ ) by spin-coating, with spinning rates between 500 and $6000 \mathrm{rpm}$, leading to an ink thickness between 130 and $400 \mathrm{~nm}$. This thickness was determined by atomic force microscopy by measuring the height of a scratch made on the ink layer. The laser conditions were then fixed to $88 \mathrm{~W}$ with a displacement of $5 \mathrm{~m} / \mathrm{min}$. Two lines, each with a width of $8 \mathrm{~mm}$, were generated across the sample by passing the laser over the sample. Half the sample was then wiped by ethyl acetate to remove all nonfixed $\mathrm{C}$ nanoparticles on the surface. A typical result is shown in Figure 3a. Four different zones were generated and analyzed, as schematized in Figure 3b. Zone 1 corresponds to the deposited layer. The intensity of the black color can be correlated to the thickness of the deposited films, as shown in Figure S3. Zone 2 corresponds to the ink layer cured with the laser, without a wiping step. The difference between zones 1 and 2 is due to nanoparticles that may have been 


\section{WILEY-VCH}

lost during laser irradiation. Zone 3 is the cured area after wiping with ethyl acetate. The difference between zones 2 and 3 provides information on the amount of $\mathrm{C}$ nanoparticles that penetrated into the polymer matrix with laser irradiation, since the nanoparticles that remain on the surface are easily removed by the solvent. Finally, zone 4 is the naked substrate. Zone 4 is used as a reference to analyze the images, providing the grayscale level of the naked substrate. All images were recorded using the same lighting conditions so that they could be compared with each other. The gray level was analyzed in each area using ImageJ software and normalized. The 0 value corresponds to complete black, and the 1 value corresponds to the white value of the PP substrate. The effects of the ink film thickness and laser power were investigated. The results are reported in Figure 3c and Figure 3d, respectively. The images of all samples are shown in Figure $\mathbf{S 3}$ and $\mathbf{S 4}$. An example of gray level extraction is given in Figure S5. For the deposited layer (zone 1 - black squares), the gray level value follows the same trend as in the transmission measurements in Figure S1, which validates this method: the intensity of the black color, as expected, increases accordingly with ink film thickness. For a lower thickness $(<250 \mathrm{~nm})$, the gray levels after laser irradiation (zone 2 - red circles) and after washing (zone 3 - blue triangles) are very similar. This result means that for this range of thicknesses, most of the $\mathrm{C}$ nanoparticles are included in the PP substrate by laser irradiation and thus remain after washing. This result corresponds to efficient permanent marking. The slight difference between zone 1 and zone 2 may be attributed to a loss of contrast when the carbon nanoparticles are inside the polymer substrate and the appearance of surface roughness. However, at a low ink thickness, the initial gray level is limited. The resulting images cannot have much contrast. An increase in the contrast can be obtained only if the ink thickness is increased. In this case, as shown in Figure 3c, the initial gray value indeed increased with thickness (zone 1), but after laser irradiation, there was no improvement in the image contrast for an initial ink thickness greater than $250 \mathrm{~nm}$. The light absorption varies from 14 to $22 \%$ for ink thicknesses in the range of 150 to $250 \mathrm{~nm}$. Consequently, we can expect the 


\section{WILEY-VCH}

temperature increase to be more pronounced with a thicker ink layer, which should favor the transfer of ink nanoparticles inside the polymer. As shown here, the results do not follow this trend. Indeed, the ink layer becomes too thick to provide efficient heating of the polymer for thicknesses greater than $250 \mathrm{~nm}$. An appreciable proportion of light may be absorbed by the surface of the ink, but the absorbed energy is not efficiently coupled to the polymer. In the end, it appears that the optimum thickness to obtain the best performances is approximately $250 \mathrm{~nm}$. These conditions correspond to a compromise in the absorption of the ink films and heat transfer to the polymer.

Another important parameter is the laser power. The effect of the laser power is reported in Figure 3d. In this set of experiments, the thickness was set to $260 \mathrm{~nm}(1500 \mathrm{rpm})$. The slight variation of the gray level in zone 1 (spin-coated film, without any irradiation) is related to the standard variation in the ink thickness from one sample to another. With laser treatment, three different regimes can be observed:

i) For the lowest powers (less than $120 \mathrm{~W}$ ), in zone 2, the gray value is almost constant, following the same trend as in zone 1. However, after washing the surface, a significant deviation from the initial gray value was observed. The marking efficiency decreases significantly, and the marking is not effective at the lowest power $(15 \mathrm{~W})$. This behavior can be explained by insufficient energy being delivered to the system by the laser, which does not allow the melting of the polymer and thus the displacement of the $\mathrm{C}$ nanoparticles.

ii) For intermediate powers (from 45 to $120 \mathrm{~W}$ ), the result is quite stable over the whole range of power. The contrast loss after washing is limited, which means that most of the C nanoparticles have been included in the polymer after laser treatment. This range of power corresponds to the optimum laser conditions for this thickness.

iii) For the highest laser power (> $120 \mathrm{~W}$ ), a decrease in the marking intensity is recorded. At the same time, fumes were observed, so this loss of contrast could be attributed to the 


\section{WILEY-VCH}

vaporization of the $\mathrm{C}$ ink due to excessive laser power. This explains why the gray value is also lower directly after laser irradiation, even without a washing step.

In the end, this study allows us to define ranges of power and thickness that are the most suitable for an efficient process. It also demonstrates that under optimized conditions, the yield of the $\mathrm{C}$ nanoparticle fixation is almost maximum.

In the following section, we consider the ink deposited by the inkjet. As shown in Figure 4, on PP substrates, the drops of ink are circular in shape. A spacing of approximately $500 \mu \mathrm{m}$ between droplets was chosen here to observe laser-induced phenomena at the scale of a single droplet. We varied the volume of the deposited drop, using volumes of 14,24 and $35 \mathrm{pL}$, to investigate the impact of the droplet volume on the marking process. As in the previous study with spin-coating, the results are compared before and after wiping with ethyl acetate to probe the displacement of the nanoparticles inside the polymer. With this configuration, lateral displacement can also be observed, which is an important parameter for inkjet printing (for resolution issues). Figure $\mathbf{4 b}$ refers to the droplet volume of $24 \mathrm{pL}$. For a lower power, we observed that the laser significantly increased the diameter of the drop. Heating of the drop by the laser decreases the viscosity of the drop, leading to spreading of the liquid over the surface. At the same time, after wiping the irradiated zone, the ink was removed, which showed that the displacement of the nanoparticles into the polymer did not occur. Interestingly, on the washed sample, the imprint of the drop is visible. This means that the melting of the polymer occurred under the ink drop, but the heating was too low to induce displacement of the nanoparticles. Increasing the laser power from $25 \mathrm{~W}$ to $50 \mathrm{~W}$ did not improve the displacement of the nanoparticles inside the polymer. The only difference between these two last conditions is the wider spreading of the ink under laser irradiation. At a power of $88 \mathrm{~W}$, laser irradiation is efficient enough to induce $\mathrm{C}$ nanoparticle fixation in the polymer. At a higher power $(120 \mathrm{~W})$, the result was similar. For smaller droplets (column a), the dependence on laser power follows a similar trend, but some differences were recorded. In particular, it 


\section{WILEY-VCH}

was observed that the spreading of the drop under laser irradiation is less pronounced. This is also consistent with the thermal spreading of the initial drop. Interestingly, at $120 \mathrm{~W}$, the increase in the diameter of the pixel is limited, and the contrast is similar after washing the surface. These conditions provide the best writing conditions to obtain the highest contrast and image resolution. As expected, when the volume of the droplet is increased, the diameter of the pixel increases without any improvement in the sensitivity of the ink to NIR irradiation. In conclusion, small droplets $(14 \mathrm{pL})$ are thus the best configuration to obtain high-quality marking.

Obviously, the results should be dependent on the polymer substrate. Indeed, the thermal parameters of the polymers strongly depend on their composition, and their surface energy will also drive the spreading of the ink droplet. The process is also highly dependent on the thickness of the deposited ink. The ink deposition and fixation processes were thus studied for the following polymers: $\mathrm{PP}\left(\mathrm{Tf}=165{ }^{\circ} \mathrm{C}\right)$, $\mathrm{PVC}\left(\mathrm{Tf}=180{ }^{\circ} \mathrm{C}\right)$, $\mathrm{ABS}\left(\mathrm{Tf}=200{ }^{\circ} \mathrm{C}\right), \mathrm{PC}$ $\left(\mathrm{Tf}=230{ }^{\circ} \mathrm{C}\right)$, and PA66 $\left(\mathrm{Tf}=258{ }^{\circ} \mathrm{C}\right)$. The results are displayed in Figure 5. First, the diameter of the drops after inkjet deposition varies greatly from one polymer to another. As previously mentioned, wetting is very limited on PP, resulting in a drop with the smallest diameter (and thus the highest height since the volume is constant). In contrast, the contact angle is zero for ABS and PC, which results in a significant spreading of the drop after deposition. For PVC and PA66, the drop spreading is intermediate. Note that at this stage, the most obvious impact of this spreading is the loss of resolution. We also emphasize that our objective was to evaluate the raw polymer surface without any chemical treatment or mechanical polishing. Spreading of the drop on ABS or PC can be modified by chemical treatment (plasma, corona or flaming). Additionally, the impact of the surface roughness of PET or PVC used here is clearly visible. The objective here is to demonstrate that marking can be effective, despite these surface defects. 


\section{WILEY-VCH}

The comparison of laser marking in these polymers highlights the importance of the fusion temperature on the efficiency of the process. Indeed, it appears that the marking process operates well for polymers with the lowest fusion temperature (less than $200{ }^{\circ} \mathrm{C}$ ) for this set of parameters. PP, PVC and ABS were indeed well marked, which is consistent with the proposed mechanism, relying on the laser-induced fusion of the polymer surface.

Some differences were noted between the polymers (Figure 5b). For PVC, a change in color was noticed after the laser treatment. Additionally, for a higher laser power, fumes were observed. They can be attributed to the thermal degradation of the polymer. However, the marking is quite efficient for this polymer with a limited loss of resolution during laser irradiation. ABS exhibited different behavior: the surface energy of the ABS surface provoked a significant spreading of the ink drop after deposition. However, laser irradiation resulted in a confined ink mark. As shown in Figure 3a, we believe that the ink thickness is too low in the periphery of the deposited drop to reach the threshold for fixing the ink into the polymer. For ABS, the fusion temperature is more important than for PP, so the threshold is higher. This point was confirmed by experiments with larger deposited drops that resulted in local burning of the polymer, as revealed by fumes. In conclusion, the process can be adapted to ABS but is not as efficient as for PP or PVC. In terms of resolutions, this process can not be considered as high-resolution one. We can indeed observe that pixels with diameters of 100 microns can be obtained in some conditions, which does not degradate too much the resolution from inkjet deposition.

However, due to their high fusion temperatures, the process is more difficult to apply to PA66, PC and PET. Indeed, we observed that the ink could be partially removed by cleaning the polymer substrate with ethyl acetate in the cases of PA66 and PET. For PC, the ink was completely removed. Interestingly, in this last case, the imprint of the drop can be clearly seen. This corresponds to a fused polymer area that is well suited to the deposited drop. As mentioned before, the fusion of the polymer surface occurred under such conditions, but the 


\section{WILEY-VCH}

temperature reached was not sufficient to induce displacement of the $\mathrm{C}$ nanoparticles. For these polymers, the laser power was increased. However, it appeared that this could not improve the results for these polymers, as the increased power resulted in a degradation of the ink. This resulted in fumes and showed that the ink was removed from the polymer surface during laser treatment.

The resistance of the ink after laser treatment was confirmed by conducting normalized aging tests. These tests are detailed in the experimental section. The ink deposited by LPT was compared to standard UV ink deposited by an inkjet on similar substrates (PP, ABS, PVC). The stability of the marking was compared under various conditions. The results are summarized in Table 1. In all cases, the LPT marking showed very good stability with no significant modification noticed after aging. The most remarkable result is associated with the olefin substrate (PP), for which the UV inkjet marking exhibits low aging properties, whereas with LPT, the marking is very stable. This result confirms the displacement process of the C nanoparticles inside the polymer.

In conclusion, we have shown that the laser polymer tattooing (LPT) process can be successfully applied to various polymer substrates with fusion temperatures below $200{ }^{\circ} \mathrm{C}$. This process is particularly interesting for marking olefin substrates such as PP. Indeed, for such polymer substrates, a stable marking could be obtained, with better aging properties than standard UV ink. The reason for this stability relies on the laser-induced process involved. The NIR laser treatment forces the ink nanoparticles to migrate into the polymer substrate. The optimization of the ink thickness and laser power allows integration of the major part of the deposited ink, so no further treatment after laser irradiation is needed. Such a process thus presents major advantages for polymer marking for potential applications in decoration, object personalization or identification and quality control. Our current works include the application of the same principle to colored inks. 


\section{WILEY-VCH}

\section{Experimental Section}

\section{Chemicals}

Black ink was prepared from a dispersion of pigment Black 7 (Aldrich, CAS Number: 133386-4) in diethylene glycol diethylether (Aldrich, CAS Number: 112-36-7). The concentration of the dye was 5 wt $\%$.

The ink was deposited by two means. i) Homogeneous thin films were prepared by spincoating using a table-top spin-coater (SPIN150i). ii) Patterns were produced by an inkjet using a SEIKO 508GS printer head. The resolution was $180 \mathrm{dpi}$, and the volume of the drops delivered was tunable between 14 and $35 \mathrm{pL}$.

NIR light from a $500 \mathrm{~W}, 808 \mathrm{~nm}$, continuous-wave laser diode (LDL500 from Laserline) was used to fix the ink. Irradiation surface was $0,16 \mathrm{~cm}^{2}$.

The printer head and laser diode were fixed, and the sample was translated by a mechanical stage with a linear speed of $5 \mathrm{~m} / \mathrm{min}$.

After laser fixation, the patterns were cleaned by firmly scrubbing the sample surfaces by hand with paper soaked with ethyl acetate.

\section{Microscopy}

Transmission electron microscopy was performed on a Philips CM200 microscope. Optical microscopy images were acquired with a binocular Olympus SZ and a Zeiss Axio Imager.M2m microscope.

\section{Aging tests:}

For the aging tests, the ink fixed by LPT was compared with standard UV ink deposited by an inkjet and stabilized by UV light. The printer was a DCS $1800 \mathrm{z}$ supplied by DirectColorSystems equipped with a UV LED $(6 \mathrm{~W}, 395 \mathrm{~nm})$. In this case, a protective UV 


\section{WILEY-VCH}

curable ink was used. It served as an adhesion promoter and protective coating. The UV ink was UV-LED IRF6 (DirectColorSystems).

Aging tests were conducted following procedures adapted from the normalized tests. The procedures are summarized in Table 1. Adherence tests were carried out after aging i) 3 days at $120{ }^{\circ} \mathrm{C}$ and ii) 4 days at $70{ }^{\circ} \mathrm{C}$ with $95 \% \mathrm{RH}$, and peeling-off tests were conducted following the ISO2409 procedure. Abrasion tests were performed following the ISO2812 procedure using a mixture of ethanol/water (60/40). Immersion tests were performed with an ethanol/water (60/40) mixture following the ISO 175 procedure.

\section{Supporting Information}

Supporting Information is available from the Wiley Online Library or from the author.

\section{Acknowledgements}

The authors thank the Fond Unique Interministeriel for its financial support (project LCOLOR3D), Moveo, Plastipolis, Vehicule du Futur and Agence National de la Recherche (grants ANR14CE26-003). Gautier Schrodj is acknowledged for DSC characterizations.

Received: ((will be filled in by the editorial staff))

Revised: ((will be filled in by the editorial staff)) Published online: ((will be filled in by the editorial staff)) 


\section{WILEY-VCH}
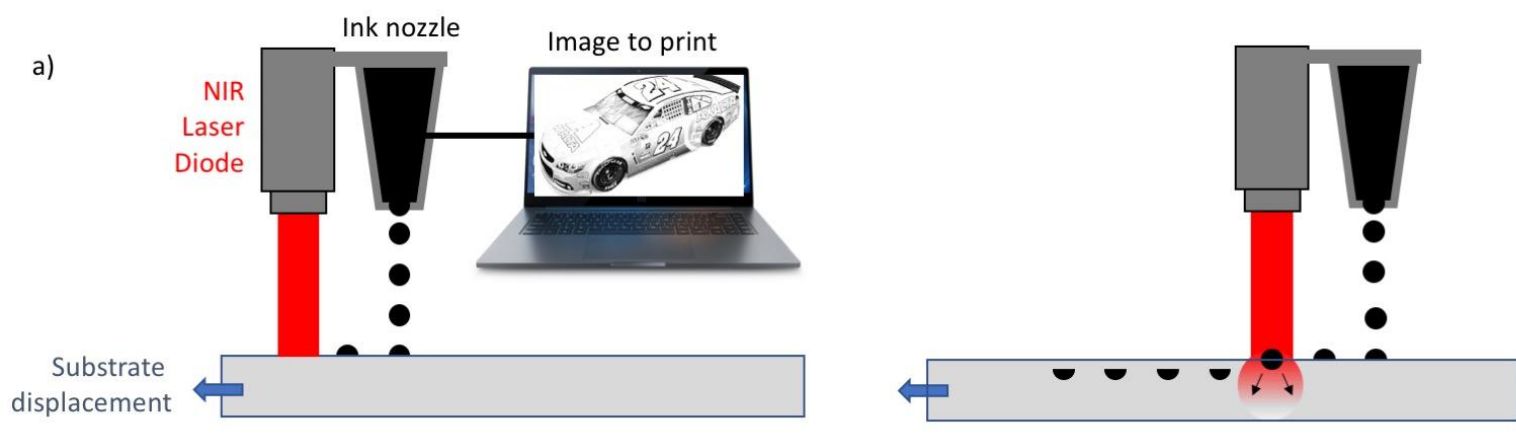

b)

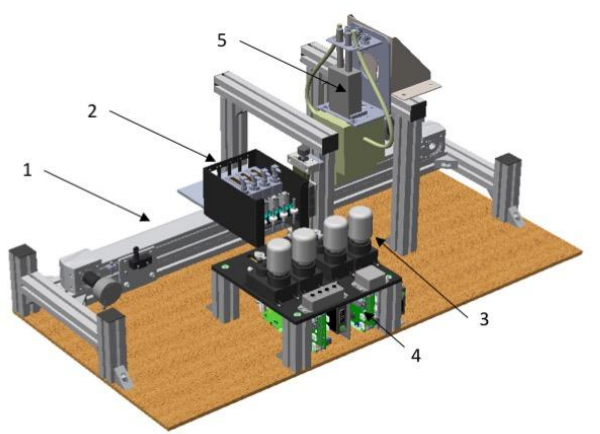

c)

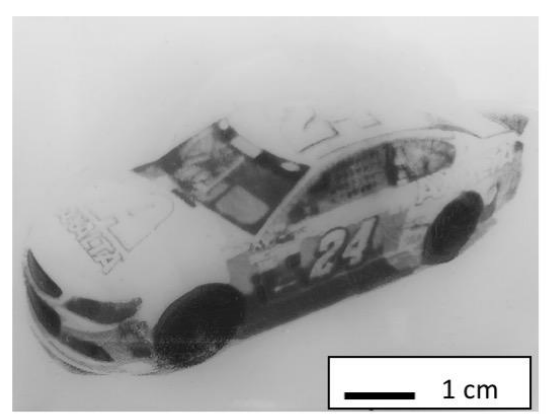

Figure 1. a) Schematic view of the polymer tattooing process showing the image to be printed. The ink is deposited by an inkjet on a moving polymer substrate. The laser is activated during the ink deposition, and laser curing occurs almost simultaneously after deposition, leading to a displacement of the ink into the polymer, as shown. b) shows a scheme of the setup including 1) the translation stage that moves the sample, 2) the inkjet heads, 3) the ink tanks, 4) the electronics driving the inkjet heads and 5) the NIR laser diode (808 nm). c) shows an example of an image performed with this setup on a polypropylene plate. 


\section{WILEY-VCH}
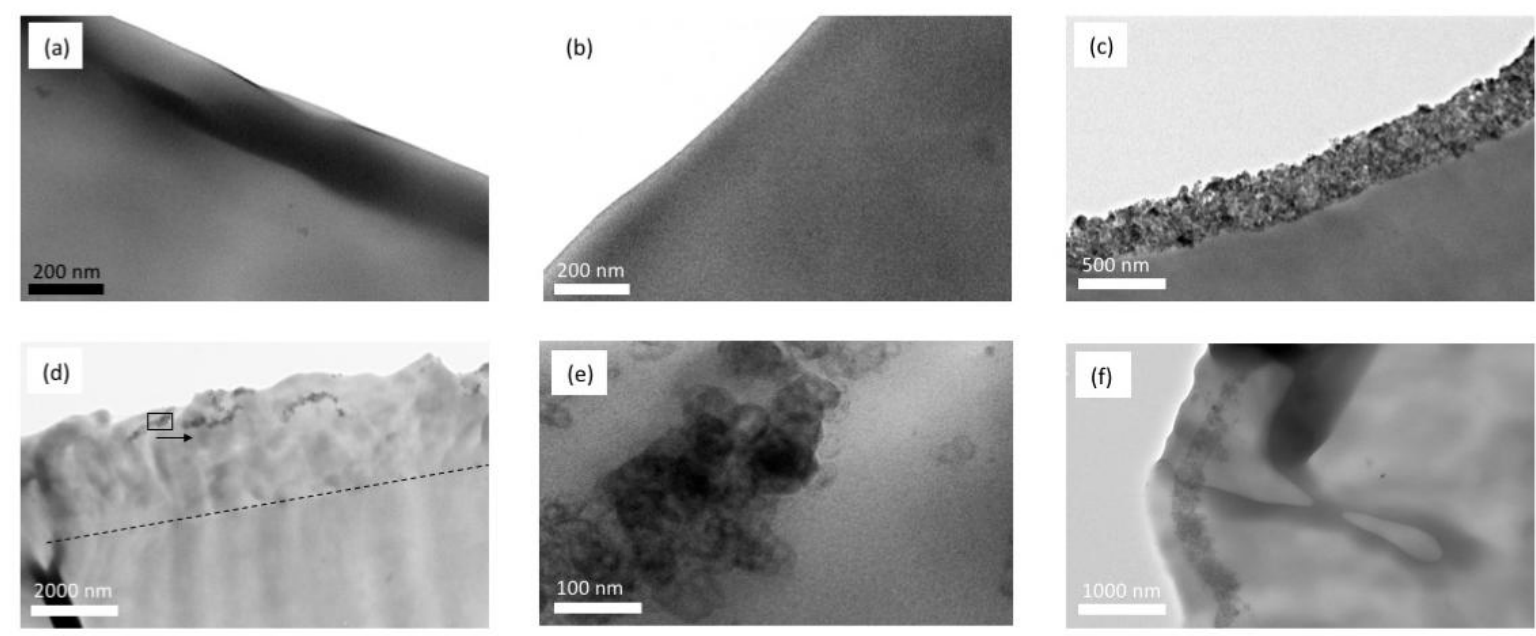

$(\mathrm{g})$

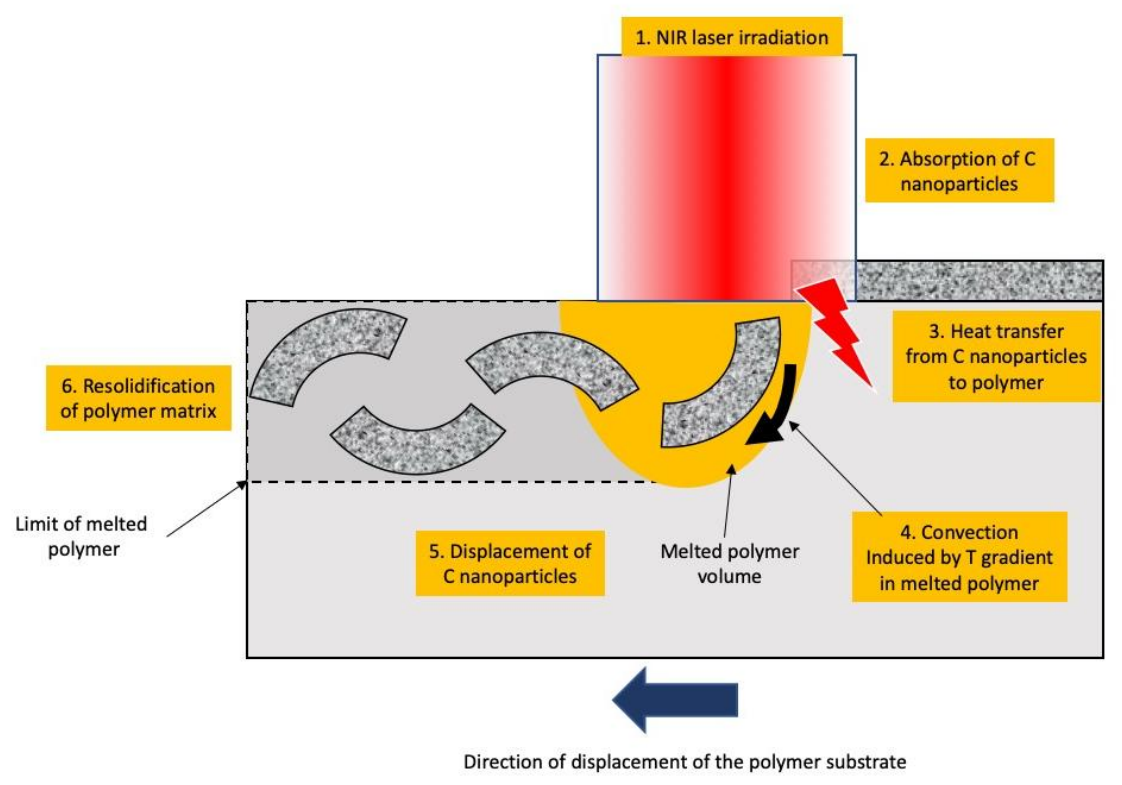

Figure 2. TEM investigation of the LPT process. a) TEM image of a polypropylene section prepared by ultramicrotomy as a reference. b) A similar surface after $808 \mathrm{~nm}$ laser irradiation (60 W, $5 \mathrm{~m} / \mathrm{min}) . \mathrm{c})$ is an of the spin-coated ink thin film. d), e) and f) show samples with ink after laser irradiation ( $60 \mathrm{~W}, 5 \mathrm{~m} / \mathrm{min})$. g) A schematic of the nanoparticle displacement under NIR laser irradiation. 


\section{WILEY-VCH}

a)

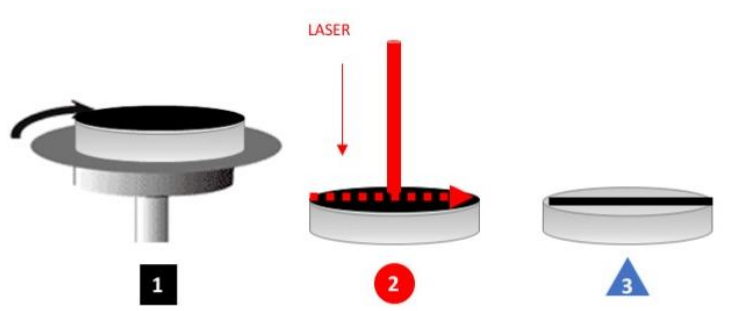

c)

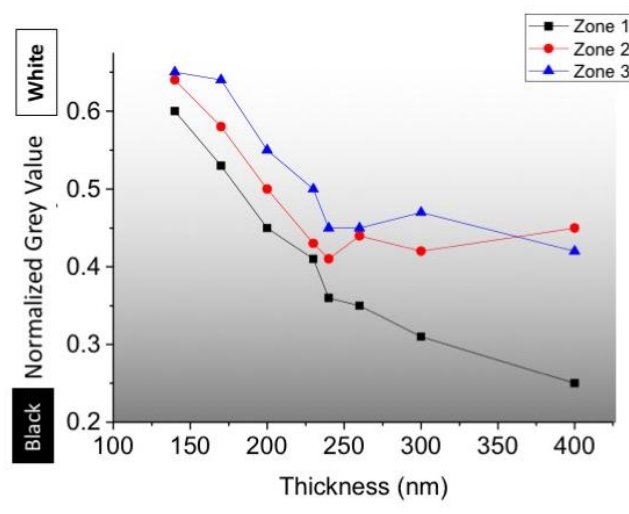

b)

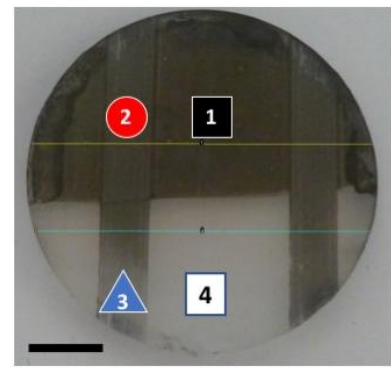

d)

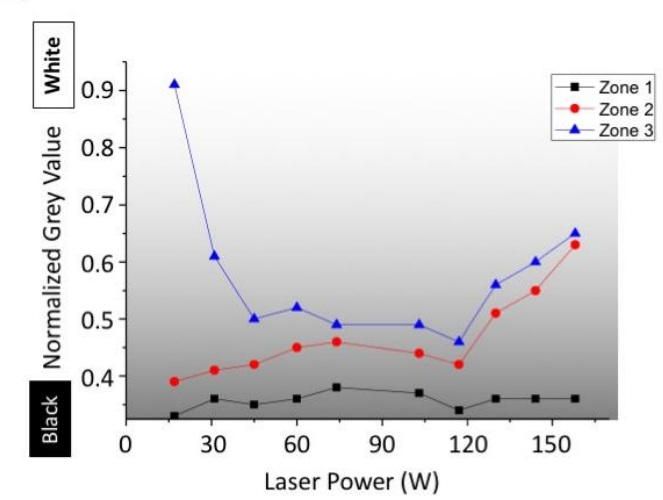

Figure 3. a) Schematic of the sample preparation for the study of the effects of ink thickness and power on the marking process, including the deposition of ink by spin-coating and laser irradiation (with a constant laser speed of $5 \mathrm{~m} / \mathrm{min}$ ). b) An image of a sample with $260 \mathrm{~nm}$ ink (1500 rpm) and laser irradiation with $\mathrm{P}=88 \mathrm{~W}$ on a PP substrate. Two laser lines were written. The top half was not washed after laser treatment. The bottom half was washed with ethyl acetate. The yellow and blue lines are the lines on which the gray level analysis was performed. The mean values in zones 1 to 4 were extracted from this image and plotted in c) and d). c) The evolution of the local grayscale with ink thickness. d) The evolution of the local grayscale with laser power. The gray values were normalized for all the images. Zones 1 to 4 correspond to 1 ) ink deposited by spin-coating, 2) laser irradiation, 3) laser irradiation and washing with ethyl acetate, and 4) naked substrate. 


\section{WILEY-VCH}

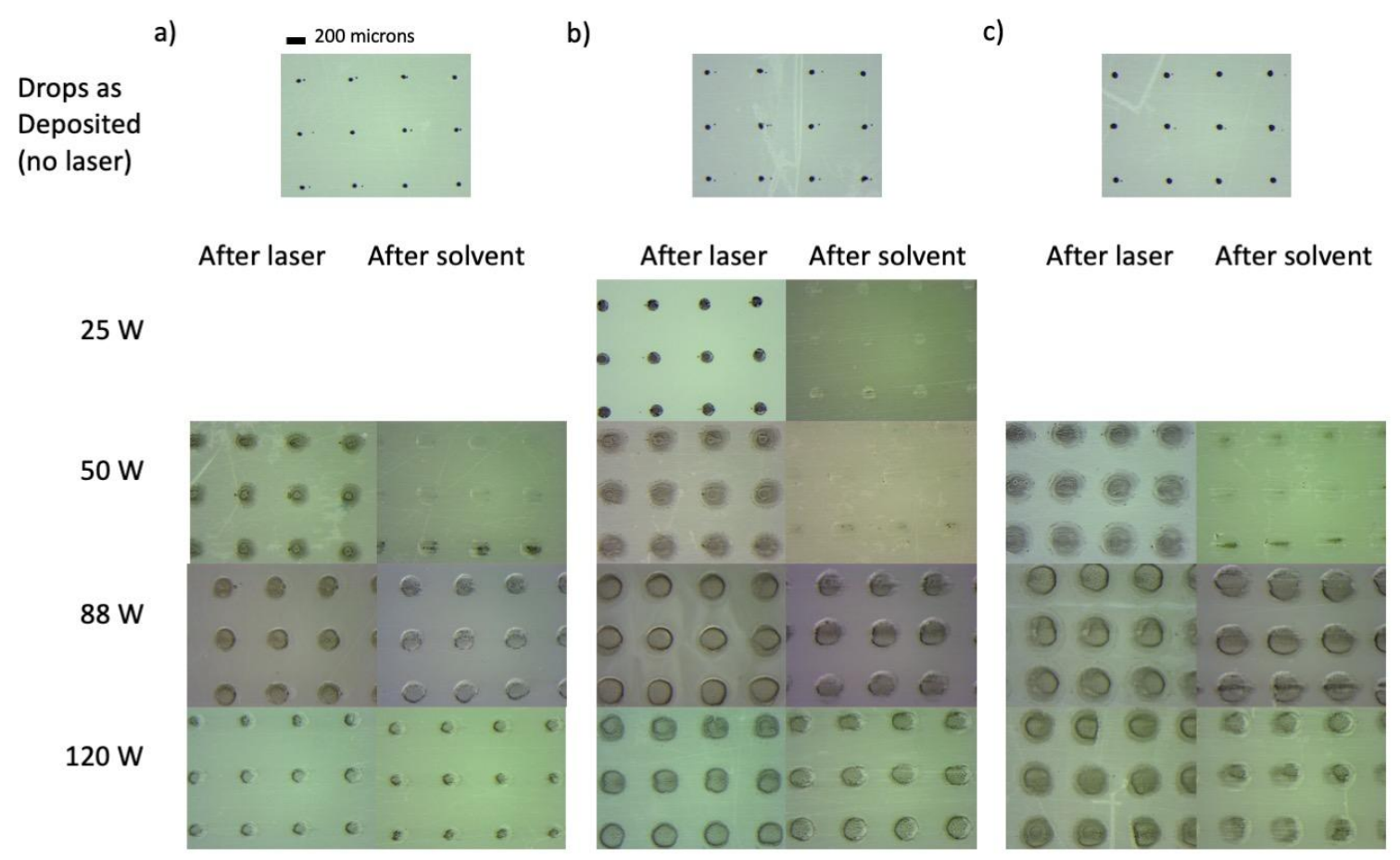

Figure 4. Optical microscopy images of the LPT process on PP substrates. a), b) and c) correspond to deposited drop volumes of 14, 24 and $35 \mathrm{pL}$. The first row shows images of the drops as deposited, and each subsequent row corresponds to different laser powers $(25,50,88$ and $120 \mathrm{~W}$ ). For each condition, images after laser irradiation and after washing with solvent (ethyl acetate) are displayed. 


\section{WILEY-VCH}

a)

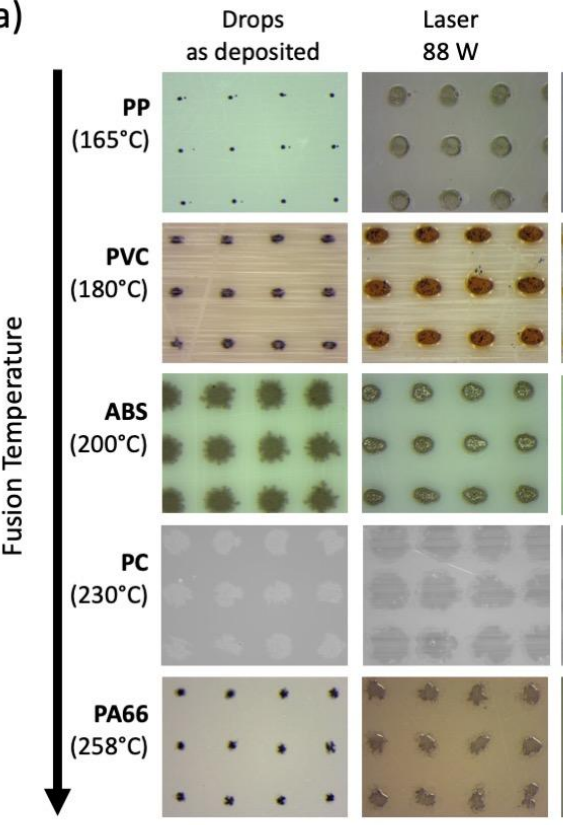

After

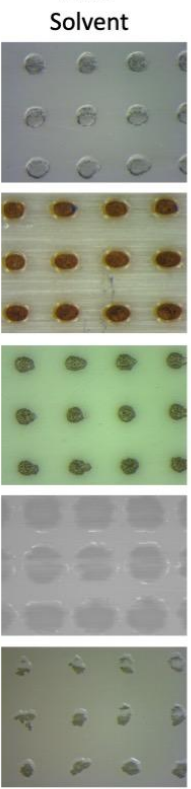

-200 microns b)

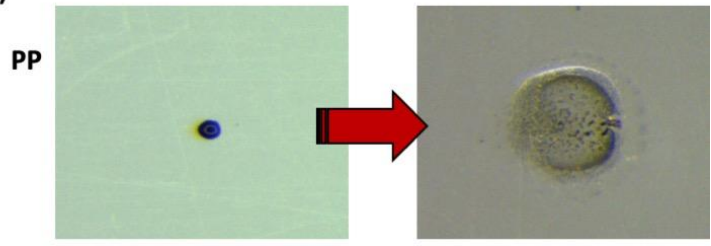

PVC

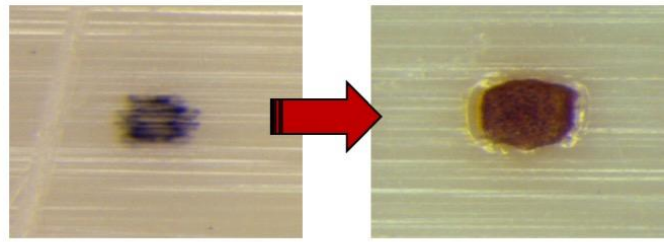

ABS

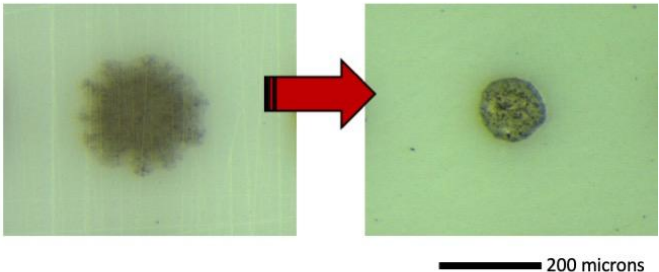

Figure 5. Comparison of LPT on various polymer substrates. a) Images of drops as deposited after laser treatment ( $88 \mathrm{~W}$ ) and after washing the surface with ethyl acetate. b) Zoomed images of the drops before and after laser irradiation (PP, PVC and ABS). 
Table 1. Comparison of ink aging after a standard UV inkjet processing and LPT for ABS, PVC, PA66 and PP.

\begin{tabular}{|c|c|c|c|c|c|c|}
\hline & \multicolumn{3}{|c|}{ UV ink } & \multicolumn{3}{|c|}{ LPT } \\
\hline Polymer & ABS & PVC & PP & ABS & PVC & $\mathbf{P P}$ \\
\hline $\begin{array}{l}\text { Adherence (DIN EN ISO } \\
\text { 2409) after aging: } \\
3 \text { days, } 120{ }^{\circ} \mathrm{C} \text { (internal } \\
\text { method, cf ISO 3248) }\end{array}$ & $\mathrm{OK}$ & $\mathrm{OK}$ & $\begin{array}{l}\text { Complete } \\
\text { peeling-off } \\
\text { ISO4 }\end{array}$ & $\mathrm{OK}$ & $\mathrm{OK}$ & $\mathrm{OK}$ \\
\hline $\begin{array}{c}\text { Adherence (DIN EN ISO } \\
\text { 2409) after aging: } \\
4 \text { days, } 70{ }^{\circ} \mathrm{C}, 95 \% \mathrm{RH} \\
\text { (internal method, cf ISO } \\
4611 \text { ) }\end{array}$ & $\mathrm{OK}$ & $\mathrm{OK}$ & Peeling-off & $\mathrm{OK}$ & $\mathrm{OK}$ & $\mathrm{OK}$ \\
\hline $\begin{array}{c}\text { Abrasion } \\
\text { Ethanol/water (60/40) } \\
\text { (internal method, cf ISO } \\
\text { 2812) }\end{array}$ & $\mathrm{OK}$ & $\mathrm{OK}$ & $\begin{array}{c}\text { Slight } \\
\text { peeling-off }\end{array}$ & OK & $\mathrm{OK}$ & $\mathrm{OK}$ \\
\hline $\begin{array}{l}\text { Immersion of ethanol/water } \\
\text { (60/40) (internal method, cf } \\
\text { ISO 175) }\end{array}$ & $\mathrm{OK}$ & $\mathrm{OK}$ & Peeling-off & OK & $\mathrm{OK}$ & $\mathrm{OK}$ \\
\hline
\end{tabular}


WILEY-VCH

TOC Figure

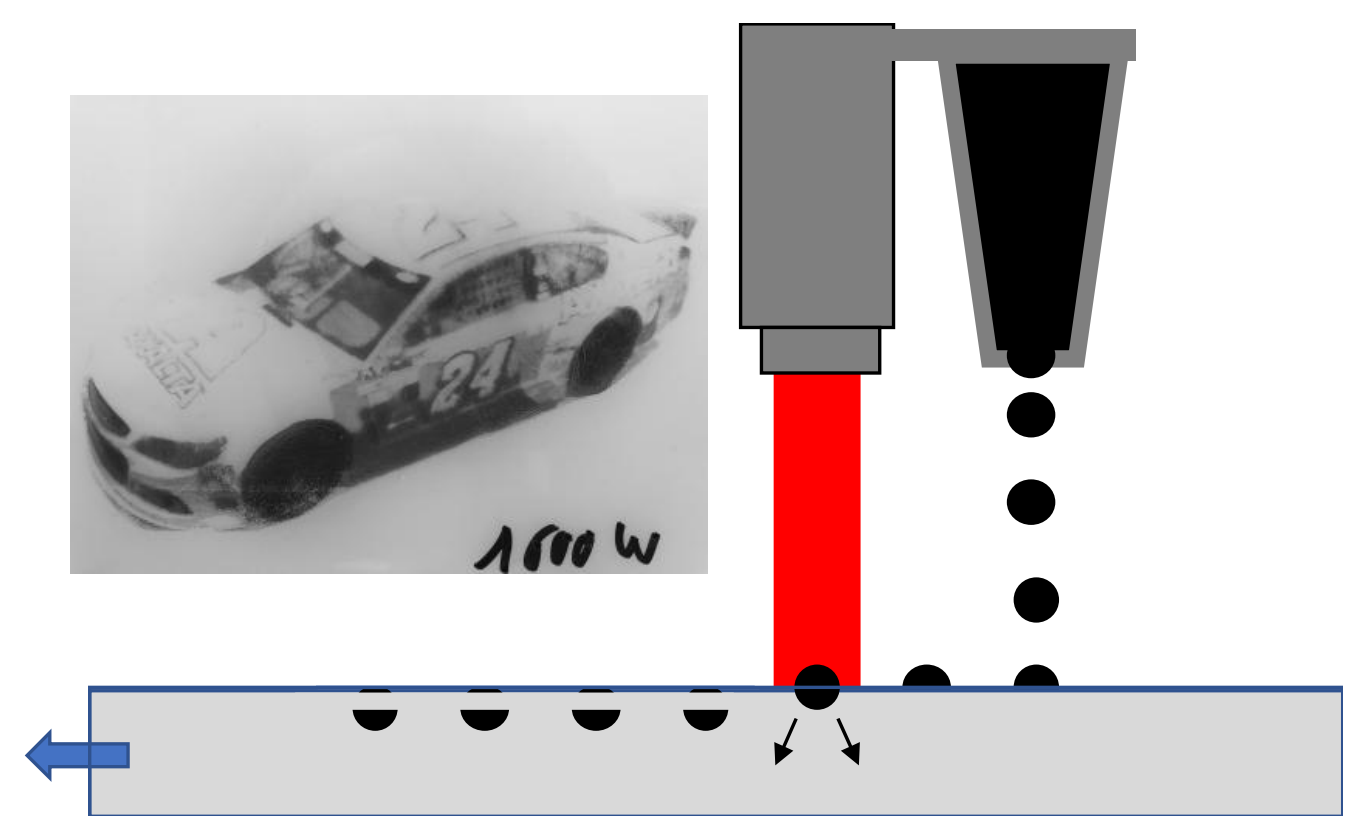




\section{WILEY-VCH}

\section{References}

${ }^{1}$ I. M. Hutchings, G. D. Martin, Inkjet Technology for Digital Fabrication, Wiley, New York, 2012.

${ }^{2}$ S. V. Murphy, A. Atala, Nat. Biotechnol. 2014, 32, 773.

${ }^{3}$ B. J. de Gans, P. C. Duineveld, U. S. Schubert, Adv. Mater. 2004, 16, 203.

${ }^{4}$ D. Bäuerle, Laser Processing and Chemistry, fourth ed., Springer Verlag, Berlin, 2011.

${ }^{5}$ F. Breaban, J. Coutouly, F. Braud, P. Deprez, Lasers Eng. 2015, 30, 1.

${ }^{6}$ Y.M. Noor, S.C. Tam, L.E.N. Lim, S. Jana, J. Mater. Process. Technol. 1994, 42, 95.

${ }^{7}$ N. Ezra, A. Arshanapalli, R. Bednarek, S. Akaishi, A.-K. Somani, J. Cosmet. Laser Ther. 2016, 18, 225.

${ }^{8}$ K. Mäntyjärvi, J. Tulonen, T. Saarnivuo, J. Porter, J.A. Karjalainen, Int. J. Mater. Form. 2008, 1, 249.

${ }^{9}$ J. Zhang, T. Zhou, L. Wen, J. Zhao, A. Zhang, ACS Appl. Mater. Interfaces 2016, 8, 1977.

${ }^{10}$ H.Y. Zheng, D. Rosseinsky, G.C. Lim, Appl. Surf. Sci. 2005, 245, 191.

${ }^{11}$ J. Chen, X. You, Z. Cao, D. Wu, C. Liu, H. Pu, Macromol. Mater. Eng. 2019, 304, 1800726.

${ }^{12}$ C. Shan Wang, J. Yueh Shieh, J. Polym. Res. 1999, 6, 149.

${ }^{13}$ A. Mohebbi, F. Mighri, A. Ajji, D. Rodrigue, Cell Polym. 2015, 34, 299.

${ }^{14}$ K. Shimamoto, Y. Sekiguchi, C. Sato Int. J. Adhes. Adhes. 2016, 67, 31.

${ }^{15}$ W. Zhong, Z. Cao, P. Qiu, D. Wu, C. Liu, H. Li, H. Zhu, ACS Appl. Mater. Interfaces, 2015, 7, 24142.

${ }^{16}$ Z. Cao, Y. Hu, Y. Lu, Y. Xiong, A. Zhou, C. Zhang, D. Wu, C. Liu, Polym. Degrad. Stab. 2017, 141, 33 .

${ }^{17}$ C. Liu, Y. Lu, Y. Xiong, Q. Zhang, A. Shi, D. Wu, H. Liang, Y. Chen, G. Liu, Z. Cao, Polymer Degradation and Stability 2018, 147, 115.

${ }^{18}$ J. Zhang, T. Zhou, L. Wen, J. Zhao, A. Zhang, ACS Appl. Mater. Interfaces 2016, 8(3), 1977. 


\section{WILEY-VCH}

${ }^{19}$ Z. Cao, Y. Hu, Q. Yu, Y. Lu, D. Wu, A. Zhou, W. Ma, Y. Xia, C. Liu, K. Loos, Adv. Engineer. Mater. 2017, 1600826

${ }^{20}$ C. Lavieja, M.J. Clemente, L. Oriol, J.I. Peña, Polymer-Plastics Technology and Engineering 2017, 56(15), 1599.

${ }^{21}$ K.S. Zelenska, S.E. Zelensky, L.V. Poperenko, K. Kanev, V. Mizeikis, V.A. Gnatyuk, Optics \& Laser Technology 2016, 76, 96.

${ }^{22}$ L. Wen, T. Zhou, J. Zhang, A. Zhang, ACS Appl. Mater. Interfaces 2016, 8(41), 28077.

${ }^{23}$ A. Piqué, R. Auyeung, J. Fitzgerald, D.B. Chrisey, Direct-write laser transfer and processing, U.S. Patent No. 7001467, 2006.

${ }^{24}$ S. V. Murphy, A. Atala, Nat. Biotechnol. 2014, 32, 773.

${ }^{25}$ F. Guillemot, A. Souquet, S. Catros, B. Guillotin, J. Lopez, M. Faucon, B. Pippenger, R. Bareille, M. Rémy, S. Bellance, P. Chabassier, J.C. Fricain, J. Amédée, Acta Biomater. 2010, 6, 2494.

${ }^{26}$ D. Munoz-Martin, C. F. Brasz, Y. Chen, M. Morales, C. B. Arnold, C. Molpeceres, Appl. Surf. Sci. 2016, 366, 389.

${ }^{27}$ C.B. Arnold, P. Serra, A. Piqué, MRS Bull. 2007, 32, 23.

${ }^{28}$ A. Piqué, H. Kim, J. Laser Micro/Nanoeng. 2014, 9, 192.

${ }^{29}$ P.A. Vetter, A. Renaud, European Patent EP1464507A1, 2004.

${ }^{30}$ S. De Iuliis, F. Migliorini, F. Cignoli, G. Zizak, Appl. Phys. B 2006, 83(3), 397.

${ }^{31}$ C. Schulz, B.F. Kock, M. Hofmann, Appl. Phys. B 2006, 83(3), 333.

32 J.J. Rulik, N.M. Mikhailenko, S.E. Zelensky, Semicond. Phys. Quant. Electron. Optoelectron. 2007, 10(2), 6.

${ }^{33}$ Fukumura, H.; Banjo, H.; Masuhara, H.; Ichinose, N.; Kawanishi, S., Applied Surface Science, 1999, 138/139, 75-83

${ }^{34}$ Fukumura, H.; Uji-i, H.; Banjo, H.; Masuhara, H.; Karnakis, D.; Ichinose, N.; Kawanishi, S.; Uchida, K.; Irie, M., Applied Surface Science, 1998, 127, 761-766

${ }^{35}$ Gery, G.; Fukumura, H.; Masuhara, H., Chemical Communications, 1998, 811-812

${ }^{36}$ Gery, G.; Fukumura, H.; Masuhara, H., Journal of Physical Chemistry B, 1997, 101, 36983705

${ }^{37}$ C.M. Jin, W. Lee, D. Kim, T. Kang, I. Choi, Small 2018, 14, 1803055. 